\title{
A Multiscale Progressive Model on Virtual Navigation
}

\author{
Xiaolong Zhang \\ College of Information Sciences and Technology, The Pennsylvania State \\ University, University Park, PA 16802, USA
}

\begin{abstract}
Navigation designs in virtual environments often draw on research findings on human navigation behaviors in the real world, in particular the landmark-route-survey spatial knowledge model. Geographers and cognitive psychologists have argued that this model is insufficient to capture the complexity of spatial cognition related to navigation. They have suggested that new theories are needed to understand the integration of various kinds of spatial knowledge and their relationship with spatial activities, such as route planning, route choosing and so on. In virtual environments, users can scale up and down the virtual space to obtain different spatial knowledge and interaction domains. Such flexibility offers an opportunity to deepen our understanding of the relationship between spatial knowledge and spatial action. This paper reports a study on how scaling in virtual environments can improve the integration of spatial knowledge and spatial action. This paper first proposes a multiscale progressive model that couples spatial knowledge and movement across scale in navigation in virtual environments. Then, the paper introduces the design of multiscale environments to support the coupling. Results of an experimental study show the benefits of the coupled spatial knowledge and movement for navigation involving subtasks at different scale levels. In addition to helping better understand the relationship between spatial knowledge and spatial action, this research also gives some insight into designs to support navigation in virtual environments as well as designs to support cross-scale spatial knowledge access in the real world.
\end{abstract}

Key words: Navigation, virtual environments, multiscale, spatial knowledge

Email address: lzhang@ist.psu.edu (Xiaolong Zhang). 


\section{Introduction}

Navigation in virtual environments is often facilitated by spatial information that users perceive visually. Spatial information could be the features of landmarks, route directions, or the overview of an environment. Different kinds of spatial information provide users with navigation guidance at different levels, from the locations of useful landmarks to a general understanding of the whole environment, and support different navigation tasks, such as planning, route selection, and destination assessment.

While research findings on human navigation behaviors in the real world have inspired navigation design in virtual environments, we should also be aware that our current knowledge of human spatial cognition is still incomplete. For example, the landmark-route-survey model, the most influential theory on navigation design in virtual environments, has been argued as being insufficient to describe the complexity of spatial cognition in navigation in the communities of behavioral geography and spatial cognition, where this model has been studied extensively. Researchers suggest that new theories are needed to deep our understanding of the process of the acquisition and integration of spatial knowledge as well as their relationship with spatial activities (Montello, 2001; Tversky, 1993; Hirtle \& Hudson, 1991; Presson \& Montello, 1988).

As spatial knowledge is usually organized as a hierarchy, in which knowledge at each layer provides people with different levels of details of spatial structures, navigation tasks are also organized in a layered manner (Timpf \& Kuhn, 2003). However, it is still unclear how hierarchical spatial knowledge and multileveled spatial tasks are related to each other. In the real world, studying the relationship between spatial knowledge and spatial tasks across scale is a challenge, because while we can read maps at different scales, our actions are

usually confined to a particular scale range from millimeters to hundreds of meter.

Virtual environments offer opportunities to examine cross-scale spatial knowledge and spatial tasks, because users can breach the scale barrier in virtual space. In this paper, we propose a multiscale progressive model to describe the relationship between spatial knowledge and spatial tasks in navigation. Under this model, navigation is understood as a series of gradually and progressively refined subtasks that require spatial knowledge and movement at different levels of spatial scale. This model argues that coupling spatial knowledge and movement and making them easily transferred across different scale levels can facilitate navigation. We also introduce a design of multiscale virtual environments to support the refinement of navigation tasks as well as the coupling and cross-scale transferring of spatial knowledge and movement. 
Section Two of this paper first reviews relevant literature on navigation in the real world and virtual environments and then presents the multiscale progressive model. Section Three describes multiscale virtual environments, which can facilitate navigation by supporting the coupling of a user's spatial perception and spatial action and the transition of this coupling across scale. This section also discusses the conceptual understanding of scale in the context of multiscale. Section Four presents an experimental study of the effectiveness of multiscale navigation. In Section Five, the paper discusses the implications of the multiscale progressive model for navigation design in virtual environments and the real world. The last section suggests some new research issues regarding multiscale navigation.

\section{A Multiscale Progressive Model for Navigation}

Navigation is a complex task. In this paper, navigation is understood as a spatial activity that is guided by visual information of the space and moves from a starting location and a destination. For example, going to a conference site from a hotel is a typical navigation activity. Navigators usually need to access spatial knowledge, either internal or external, in navigating. They also need to control their movement to achieve navigation goals. The proposed multiscale progressive model is an effort to improve our understanding of both the navigation process and the relationship between spatial knowledge and movement. This model has its roots in research on mental and physical processes of navigation in the real world and in virtual environments.

\subsection{Navigation in the Real World}

Research issues on navigation are highly diversified. Our interest is in helping users easily understand environments and move around in large virtual spaces. Thus, the literature reviewed focuses on the acquisition and application of spatial knowledge.

Spatial knowledge is usually considered to be in three forms: landmark knowledge, route knowledge, and survey knowledge (Piaget \& Inhelder, 1967; Hart \& Moore, 1973). Three forms of spatial knowledge provide different levels of abstraction of space. Object representations at lower levels are grouped as object clusters that become components of higher levels (Kosslyn et al., 1978). Spatial knowledge helps people build their internal spatial representations of external environments, or cognitive maps (Tolman, 1948), which are often constructed as hierarchies, grouping different features of space (Stevens \& Coupe, 1978) and complex spatial layouts (Hommel et al., 2000) into levels. Such lay- 
ered structures allow people to effectively access necessary spatial knowledge in spatial problem solving (Stevens \& Coupe, 1978; McNamara et al., 1989). For example, Chase (1983) showed that taxi drivers used different kinds of spatial knowledge in driving: coarser spatial information when driving from region to region and finer information when driving within the neighborhood of destination.

It has been argued that the understanding of new environments is built on a sequence following landmarks, routes, and configurations (Siegel \& White, 1975), but such a traditional view on spatial knowledge and cognitive maps has been challenged. Researchers have argued that the acquisition of spatial knowledge and the construction of cognitive maps involve more complicated processes and techniques (Montello, 2001; Tversky, 1993; Hirtle \& Hudson, 1991; Presson \& Montello, 1988).

Spatial knowledge is critical to wayfinding in navigation, which refers to finding a way to a particular location (Downs \& Stea, 1973). In wayfinding, people need spatial knowledge about an environment (Golledge, 1999), and often rely on aids like maps as external artifacts for and cognitive interfaces to the external environment (Bagrow, 1985; Barkowsky \& Freksa, 1997). Using maps, however, requires skills to establish object correspondence between maps and the world and to align maps with the environment (Newcombe \& Huttenlocher, 2000). These skills could be difficult to learn and apply because people tend to encode spatial knowledge from maps and from direct environmental exposures differently (Evans \& Pezdek, 1980; Levine et al., 1985; Thorndyke \& Hayes-Roth, 1982).

Navigation can become easy if environments are well-structured and organized by following certain design patterns (Lynch, 1960), or even a pattern language, in which smaller patterns are nested in larger ones (Alexander et al., 1977). Layered structures can help people better understand large environments because they allow the deployment of scarce cognitive resources-memory and attention-toward tasks at proper scale levels (Ahl \& Allen, 1996).

\subsection{Navigation Design in Virtual Environments}

Spatial cognition in virtual environments has been found to be similar to that in the real world (Ruddle et al., 1997; Wilson et al., 1997; Witmer et al., 1996), so researchers have drawn on the results of research on navigation in the real world to support wayfinding in virtual environments. Various designs have been proposed to support access to spatial knowledge. One often-seen technique for acquiring survey knowledge is to place a $2 \mathrm{D}$ overview map on top of a $3 \mathrm{D}$ view. However, aligning $2 \mathrm{D}$ maps with $3 \mathrm{D}$ environments is still a 
problem (May et al., 1995); tools like automatic alignment are needed (Darken \& Cevik, 1999). Overviews can also be in 3D, such as a miniature model of the world (Stoakley et al., 1995; LaViola et al., 2001). Compared with 2D maps, $3 \mathrm{D}$ overviews tend to be easier to use because of their similarity to the real world (Liben, 2001). Design efforts have also been made to help acquire landmark knowledge (Vinson, 1999). Route knowledge in virtual environments still largely relies on visual information such as key landmarks (Elvins et al., 1997), given that there is less involvement of body movement in virtual navigation.

However, simply providing users with different kinds of spatial information seems insufficient. Colle and Reid (1998) showed that even with direct access to landmark knowledge and route knowledge, people's global understanding of a space is confined by local spatial settings, such as walls of rooms. This result, called the "Room Effect", provides empirical evidence for the argument by some geographers that spatial knowledge integration may not be a staged process, as traditionally thought, and may involve more complicated processes and techniques (Montello, 2001; Tversky, 1993; Hirtle \& Hudson, 1991; Presson \& Montello, 1988). Thus, there is a need to study the integration processes and to design new tools to support such processes.

In virtual environments, travel control is as important as spatial knowledge access (Bowman, et al., 1997). Travel moves a navigator from one place to another, changing the position of the viewpoint. While wayfinding emphasizes the mental processes of navigation planning, travel concerns physical processes to execute navigation plans. Mackinlay et al. (1990) argued that one big challenge for travel control is to balance movement speed and accuracy. In some situations, users can rely on system tools like system-walking (Hanson et al., 1997), teleportation, and special trajectory functions (Mackinlay et al., 1990). However, users often need control over their travel during the whole navigation process, including when to start and stop movement, where to go, and how to go. Moreover, in addition to move around to obtain spatial information at the same scale level, users also need to access spatial information across scale. Such cross-space and cross-scale exploration movement is of interest to this research.

Some efforts have been made to integrate wayfinding and travel. For example, the Place Representations technique (Pierce \& Pausch, 2004) incorporates spatial information into a hierarchical structure so that users can obtain spatial information with different levels of details in travel. Tan et al. (2001) adapted the presentation of spatial information to a user's flying speed.

Some models on navigation tasks in virtual environments have been proposed. Darken and Sibert (1996) recognized three navigation tasks for different purposes: naïve search, primed search, and exploration, but their model did not describe how these three mutually exclusive tasks are related in navigation. 
The navigation framework by Jul and Furnas (1997) identified important factors in navigation such as forming navigation goals, perceiving spatial knowledge, moving, and accessing navigation results. A staged process model by Chen and Stanney (1999) divided navigation tasks into the three subtasks of cognitive mapping, decision making, and decision execution. Both models suggested navigation as a process including tasks at multiple levels, but neither elaborated how subtasks were formed and how they evolved.

\subsection{A Multiscale Progressive Model of Navigation}

The multiscale progressive model proposed here is targeted to navigation activities in which the spatial location of a target, or more specifically its coordinates, is unknown and a navigator needs to explore the space based on other kinds of descriptive information, such as shape, color, appearances and so on, about the target. With the knowledge of the coordinates of a target, system-driven techniques like logarithmic movement (Mackinlay et al., 1990) and the Image-Plane-Manipulation (Pierce et al., 1997) can be used. Users can even use teleportation tools to get there more efficiently.

When spatial coordinates are unknown and the only available information is other descriptive information, users need to explore the space and find the target based on the descriptions of the target. Descriptive information of a target is often easy to be perceived by users, but difficult to be processed by computers. While it might not be a challenge for a user to locate a building with a specific shape, for example, it is often a challenge for a computer system to do so unless the shape information is embedded in the $3 \mathrm{D}$ model of the building. It would be ideal if a $3 \mathrm{D}$ environment can contain such descriptive information in all individual 3D models. It is not realistic, however, because embedding such information in a 3D system would dramatically increase the amount of data the system has to process. Also, even if we can find a way to deal with data processing issues, it is still not guaranteed that descriptive information the system has is comprehensive enough to meet the needs for descriptive information by different users. Therefore, it might be better to improve our understanding of how users explore space through self navigation to find a target with descriptive information.

A user's knowledge about a target may not be specific enough to pinpoint the target. In an environment with complicated objects, the user often has to use descriptive information in finding the target. For example, when people ask for the direction of a particular location, the answer is usually not the coordinates of the location. Rather, what is often given are descriptions on attributes that human beings can easily perceive, such as color, shape, or surrounding spatial objects. We can also find another example in an anti- 
terrorist scenario, in which a suspect who placed a bomb and later was caught may only tell the law enforcement a rough location of the bomb, such as in a car parked near an entrance of a gray building in the financial district, rather than exact spatial coordinates. Under such situations, exploring space through navigation is needed to locate the target.

The multiscale progressive model is an effort to understand such explorationoriented navigation activities. This model sees exploration-oriented navigation as an evolving process in which navigation tasks are gradually refined. Consider a scenario of going to a conference in an unfamiliar city. We usually decompose the overall goal, which is to get to the conference room, into a sequence of subgoals: going to the neighborhood of the conference place, finding the conference place, and locating the conference room. The overall navigation task is a set of subtasks at different levels. Each subtask involves such activities as subgoal planning, moving, perceiving the environment, and assessing the movement results (Figure 1a). The accomplishment of a subtask is the condition of the pursuit of the subtask at the next level (Figure 1b).

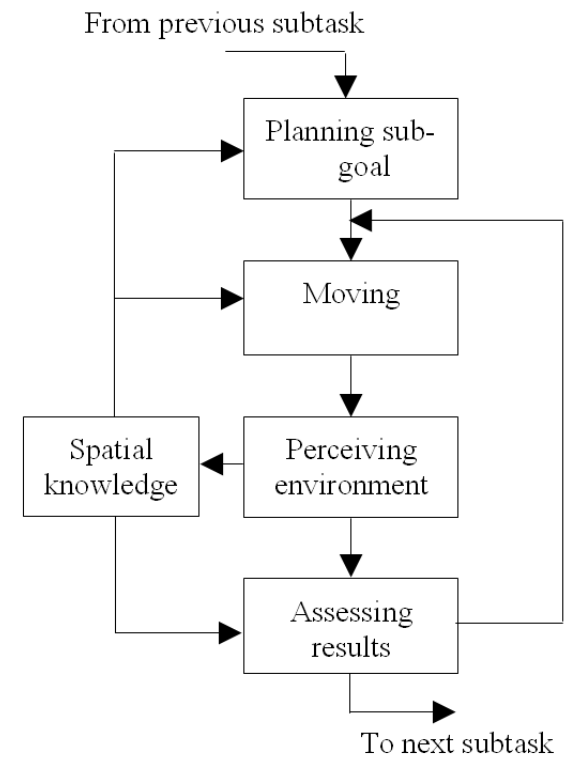

(a)

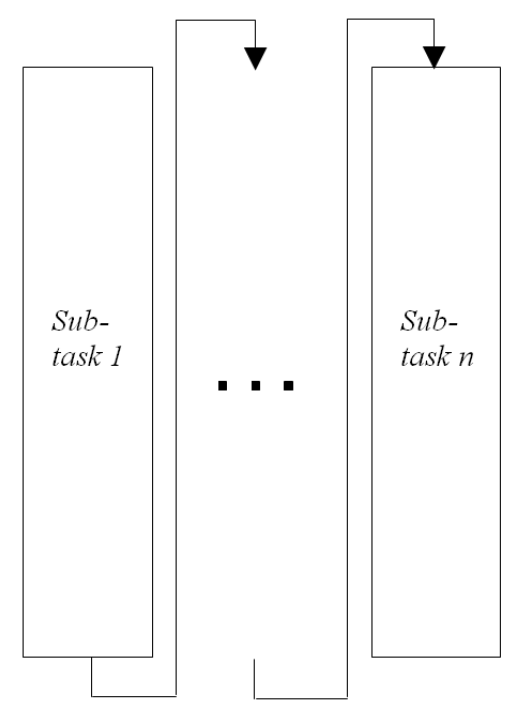

(b)

Figure 1. A multiscale progressive model on navigation: a) is the subtask unit that shows the activities needed to complete a subtask; b) indicates the decomposition of the overall navigation task into a set of subtasks, each of which is a subtask unit.

Completing a subtask requires spatial knowledge. We need spatial knowledge for goal planning, movement guidance, and navigation result assessment. During moving, we perceive the environment and update our spatial knowledge. If the subgoal has not been achieved, further movement would be needed. Otherwise, we shift navigation to the subtask at the next level. For a particular subtask, we will need spatial knowledge appropriate to this task. When we complete one subtask and move to the subtask at another level, required spa- 
tial knowledge also changes. For example, to find the conference place, we need a map of its neighborhood, but after we have located the conference place, we will need a floor map to help us find the conference room.

What has been changed with the level of subtasks also includes action accuracy. Different subtasks may have different requirements for movement accuracy. Being in the neighborhood of the conference place needs only be accurate to tens or even hundreds of meters, but finding the door to the conference room requires position accuracy at the level of the meter. Different movement accuracies imply that people can choose different movement speeds for different subtasks to achieve efficiency, as long as movement accuracy can satisfy the error tolerance for each subtask.

Spatial knowledge and movement at each level need to be comparable. Spatial knowledge guides movement, and movement updates spatial knowledge. Unmatched spatial knowledge and movement may make it difficult for people to plan their actions and assess the results of the actions. For example, a map of the conference place area with a resolution of tens or hundreds of meters would be suitable for locating the conference place, but not for finding the conference room, which requires a floor map of the conference place with a resolution at the meter level.

Such cross-scale transition should be smooth so that users can easily see how objects at different scales are related to each other. This will allow users to establish the connection between objects and to align spatial structures easily. For example, in order to be a specific place of a building, a user can first choose a scale level to approach the target building quickly, and then change to another scale level to adjust the final location accurately. A smooth transition of spatial knowledge between these two different scales makes the targeted building always visible. The object constancy in the smooth transition provides the user with the frame of reference in navigation. The user can clearly see how new spatial information is related to previous one and easily know where to go next for the following subtask.

The granularity transformation model of wayfinding by Timpf and Kuhn (2003) saw wayfinding in the real world as a hierarchical process based on the differently-scaled maps. Their model largely focused on the cognitive aspect of navigation activities in goal planning, strategy choosing, and moving. The progressive model proposed in this paper, by considering both spatial knowledge and spatial actions-movement, emphasizes that navigation is an evolving process with gradually refined subgoals, and argues the necessity of coupling spatial knowledge and movement as well as easily transferring them across different subgoals. 


\section{Multiscale Virtual Environments}

It would be helpful if users can control the process of gradually refining navigation tasks by having tools to couple spatial knowledge and locomotion speed together and transfer them to different scale levels. One way to address this issue is to integrate multiscale technology into virtual environments.

\subsection{Multiscale Technology}

The term "multiscale" was coined by Perlin and Fox (1993). Inspired by the concept of scale and scaling operations in 3D graphics, they introduced scalable workspace into 2D user interfaces, and designed the first 2D multiscale user interface, Pad. Pad and its successor, Pad++ (Bederson \& Hollan, 1994), allow users to manipulate the rendered size of the workspace to get multiple display magnifications and action domains. Scaling up the workspace, users can see small things and make small movements. Scaling down the workspace, they see huge things and make big movements. Thus, users can interact with space at different scale levels in multiscale user interfaces. More precisely, scale refers to interaction scale, a parameter that coordinates a set of size-related parameters for user interactions, such as eye-level, navigation speed, and manipulation distance. This interaction scale can be directly controlled by users. Changing interaction scale is changing these size-related interaction parameters together (Zhang \& Furnas, 2005a, 2005b). Integrating multiscale tools into 3D virtual environments allows users to control the scale level at which they interact with virtual worlds and to get different spatial knowledge and movement capabilities.

Some research shows that multiscale technology has the potential to improve navigation in large information worlds. Its benefits include shortening the navigation path (Furnas \& Bederson, 1995) and improving viewpoint control in the workspace (Guiard et al., 1999). Theoretically, easy access to information of large structures at different levels can help navigation (Bederson \& Hollan, 1994), but research on this issue produced inconsistent results (Combs \& Bederson, 1999; Ghosh \& Shneiderman, 1999). Hornbæk et al. (2002) argued that the inconsistency may be due to different research designs, rather than multiscale technology itself.

Large space in multiscale user interfaces also causes problems. Users may be trapped in places where no visual information is available for navigation at all. This "desert fog" problem (Jul \& Furnas, 1998) could severely impede wayfinding. 


\subsection{Multiscale Virtual Environments (mVEs)}

Extending multiscale technology into 3D worlds creates mVEs. In mVEs, users can directly use scaling tools to shrink the world to see the big picture and increase their movement speed to approach a target quickly and coarsely, and then magnify the world to observe the fine details and increase their movement accuracy to precisely position themselves at their destinations. With scaling tools, users can also access various kinds of spatial information and integrate appropriate spatial information with necessary spatial tasks.

Of course, integrating scaling techniques into virtual environments is not totally new, and many research projects have considered the scale factor in interaction design (Robinett \& Holloway, 1992; Mapes \& Moshell, 1995; Mine et al., 1997; Ware \& Fleet, 1997). Multiscale techniques extend scaling operations in virtual environments in several ways. First, in multiscale environments, scaling is treated as a basic and first-class spatial parameter in the context of user in-

teraction. Scale is one of three fundamental operations in 3D graphics matrix transformation. (The other two are translation and rotation.) However, scale in 3D graphics usually concerns the size of individual objects in modeling. At the level of user interaction, while the translation and rotation of user's viewpoint are always supported in navigation design and can be directly controlled by users, viewing scale is often not controllable. Some designs provided users with scaled-down overviews (Stoakley et al., 1995; LaViola et al., 2001), but these designs let systems, rather than users, control at what scale interaction should happen and how to reach that scale. Thus, compared to translating and rotating in view control, scaling is treated more like a second-class parameter in most conventional 3D environment. Multiscale environments elevates scaling to the same level as translating and rotating. Users can fully control at what scale levels they want to interact with the world. For example, a user can be a giant at one moment to enjoy a global view of a city and a great action domain, and be a small ant at another moment to get a local view of building details and precise action.

Second, scaling in multiscale environments provide users with dynamic and smooth view transition across space and scale, and users will see the continuous change of objects in not only their geometric sizes, but also their different semantic appearances across scale. Users can access richer spatial information at different levels, from global to local, and in different forms, from 2D map overviews to 3D immersive environments. Of course, 3D animation, as one of features of virtual environments, can provide users with dynamic and smooth view transition across space. What we try to emphasize here is that multiscale virtual environments also support dynamic and smooth view transition between scale-dependent semantic representations across scale. When different semantic representations are used at different scales, the change be- 
tween two representations may confuse users. For example, when users are provided with two different representations of the same city for wayfinding a detailed 3D view for route evaluation at the local scale and a schematized perspective map for route planning at the global level, the switch from one representation to another may distract users from their primary tasks because of the significant difference between objects in two representations. Thus, it is important to make the view transition between different representations easily comprehensible in multiscale environments. Technical details on the design and implementation of scale-dependent semantic representations can be found in our other papers (Zhang \& Furnas, 2005a; Zhang, 2007).

Furthermore, multiscale design considers both action and perception. Users' perception and action are yoked under one interaction parameter, interaction scale, which can be directly controlled by users. It should be pointed out that perception and action discussed here concern the whole environment, rather than individual objects in the environment, because scaling in multiscale virtual environments refers to an activity to enlarge or shrink the environment as a whole. Thus, when an environment is scaled up and down, the relative size between a user and the environment is changed. Consequently, what the user can see about the environment and do with it differs from scale to scale. The scale factor changes both the perception and action capabilities.

Yoking these two parameters is important if a user wants to interact with space across scale. Figure 2 shows images of the same environments at different scale levels. While Figure $2 \mathrm{a}$ gives users a view of a specific building, Figure $2 \mathrm{~b}$ to $2 \mathrm{~d}$ allow users to see the building clusters, a district, and even the whole city respectively. Users can control the view scale directly and dynamically, just as they control their observation locations.

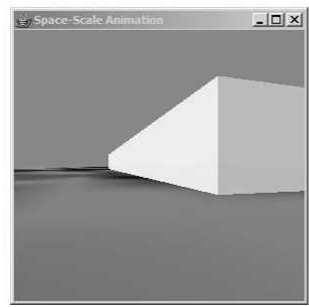

(a)

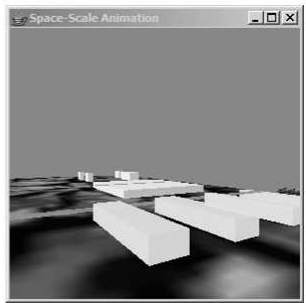

(b)

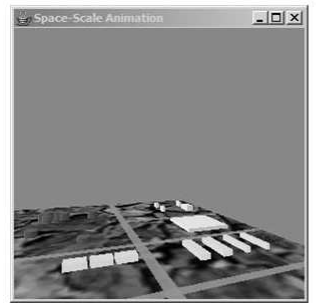

(c)

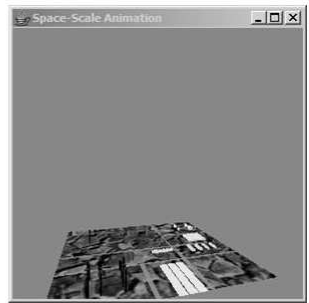

(d)

Figure 2. Views of a city at different scale levels: (a) is at the street level; (b) is at the level of neighborhood; (c) is at the district level; and (d) is at the city level.

At any given scale, users possess action capabilities appropriate to that scale. For example, when a user is interacting with the world at the street level seen in Figure 2a, the user's movement scale should be small enough so that it is easy to examine detailed spatial information on the street, as seen in Figure 3. When the user moves to the level of the city, the movement should be large enough to allow quickly browsing the city, as seen in Figure 4. Only with such 
spatial knowledge and spatial action coupling can the user achieve navigation goals at different scales. Unmatched spatial knowledge and spatial task would either get the user lost due to the significant view change, if the user has a street-level view but a city-level movement, or demand tremendous efforts to navigate, if the user has a city-level view but a street-level movement.

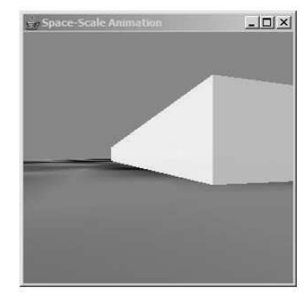

(a)

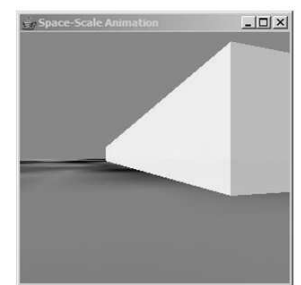

(b)

Figure 3. Movement at the street level: (a) and (b) are views before and after a one-step movement. The small step allows users to change their views of the street slightly so that they can control their movement precisely.

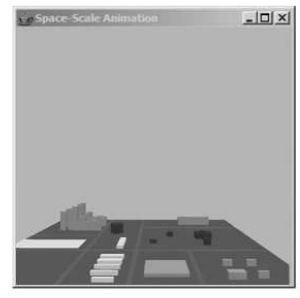

(a)

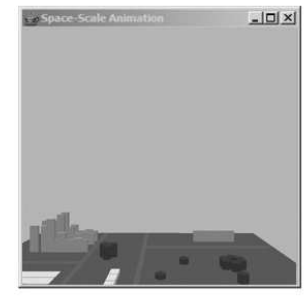

(b)

Figure 4. Movement at the city level: (a) and (b) are views before and after a one-step movement. The big movement step allows users to change their views of the city dramatically so that they can quickly browse the global information of the city.

It should be noted that yoking perception and action under one interaction parameter is a design to support user interaction with the whole environment. When interaction is between users and individual objects, rather than the whole space, users may not want to yoke perception and action together. For example, in some applications such as Computer-Aided Design systems or virtual environment modeling systems, users may want to examine the objects of interest by scaling them up and down, but without affecting their action domains on other objects and space. Scaling in this context can be more accurately described as an operation to resize individual objects, rather than the whole environment. In multiscale environments, such resizing operations can co-exist with scaling operations, which affect the whole environment, given their different purposes in user interaction.

Yoking perception and action together requires spatial information, which is related to perception, and movement, which concerns action, to be coupled across scale. To support the coupling and cross-scale control of spatial information and movement, we used an avatar metaphor in the design of mVEs. 
Users often interact with virtual space through their virtual representations, or avatars, in virtual environments. An avatar usually appears as a virtual human with virtual eyes, virtual arms, and virtual legs. In mVEs, these body parts are used to set a user's interaction parameter: virtual eyes to set the viewpoint position and virtual legs to define movement step size. Changing interaction scales means enlarging or shrinking the size of a user's avatar relative to the virtual world (or equivalently, shrinking or magnifying the world size relative to the avatar), and consequently rescaling the values of relevant interaction parameters together.

Two issues should be pointed out here. First, the avatar metaphor is not the only way to describe scalable interaction parameters. Some scaling tools for manipulation did not use avatars (Mine et la., 1997; Pierce et al., 1997). However, these tools usually focused on just one interaction parameter without considering others. In mVEs, an avatar metaphor helps tie interaction parameters (e.g., viewing position and step size) together so that users can get spatial knowledge coupled with movement.

Second, multiscale tools in virtual environments share some features with the level of detail (LOD) techniques. They both visualize the same structure with different geometric objects according to a particular interaction parameter: scale in multiscale virtual environments and distance in regular LOD techniques. However, the use of different geometric objects in multiscale virtual environments and LOD techniques have different purposes. While computation efficiency is the primary concern of most LOD techniques (Puppo \& Scopigno, 1997), user needs in interaction are what multiscale virtual environments really care about. Data abstraction in LOD rendering exploits the incapability of human users to recognize fine details of distant objects and uses simpler geometries to represent more complex models, while multiscale virtual environments incorporate different models by considering users' different tasks and providing different kinds of spatial knowledge with details appropriate to these tasks. For example, in traditional LOD techniques, a building is usually rendered as a simple box at distance. In multiscale virtual environment, however, a distant building would be rendered with a text label if it could provide navigation guidance as survey knowledge.

\subsection{Navigation in Multiscale Virtual Environments}

The association of the viewpoint position and step size in avatars ties a user's spatial knowledge and movement together. Users can simply change the scale between their avatars and the world to obtain proper spatial knowledge and movement speed and accuracy. Goal planning, moving, and plan assessing can be conducted based on the same kind of spatial knowledge. When a subgoal 
is reached, users can rescale the world to the level appropriate for the next subtask. In this process, users do not need other tools such as maps to access survey knowledge for planning, as people always do in the real world, and can easily adjust their movement speed and accuracy according to the level of subgoals.

Another benefit of multiscale navigation is that it may further improve the understanding of space. In mVEs, users can become a giant to see the whole space and move quickly from one region to another. They can then obtain survey knowledge through direct exploration. In the real world, although overviews of a city can be obtained in places like high buildings or airplanes, people can hardly experience the city at this level through direct body movement.

Researchers begin to notice such potentials of multiscale technology in support of user navigation in virtual environments. A recent study by Kopper, et al. (2006) shows that multiscale tools can help users to navigate through large, cross-scale virtual environments. While this study focuses on helping users maintain spatial orientation in navigation, our research is about relationship between spatial knowledge and locomotion control.

\subsection{Interpretations of Scale in Multiscale}

The term "scale" could mean very different things under different circumstances (Lam \& Quattrochi, 1992; Reitsma \& Bittner, 2003; Albrect \& Car, 1999; Pereira, 2002; Montello, 2001). Scale in multiscale emphasizes the spatial relationship between the space and what users can perceive and act upon rather than the presentation of spatial information. The scale in multiscale virtual environments is close to Montello's interpretation of scale (1993) as a measure of the relationship between the perceived space and human, but with a different understanding of the relationship between scale and human users/actors. In Montello's notion of scale, human actors, as passive perceivers in an environment with a given scale, cannot do anything about the scale. In mVEs, however, users can actively manipulate the scale at which they see and act, as seen in Figure 2 and 3.

It is this involvement of users in scaling control that distinguishes scale in multiscale from other interpretations of scale. Direct and active scale control could improve users' understanding of space. It allows users to easily obtain different kinds of spatial knowledge and see the transformation of and relationship between spatial structures at different scales. Thus, scaling is a process of active spatial exploration, which can help better integrate users into environments (Hutchins, 1995) and improve their knowledge about the space (Cohen \& Cohen, 1985; Péruch et al., 1995). 


\section{Experimental Study}

To deepen our understanding of the multiscale progressive model, we conducted an experiment to study how progressively-refined spatial knowledge and spatial action may facilitate navigation and whether coupling spatial knowledge and movement across scale may improve navigation performances. The test environment was a desktop mVE implemented with Java 3D.

\subsection{Experimental Design}

The experiment was a " 2 X $2+1$ " design. The 2 X 2 part crossed fixed eye level/changeable eye level with fixed step size/changeable step size (Table 1). Here, "fixed" means a parameter could not be modified by users, and those "changeable" treatments allow subjects to change corresponding parameters. Eye level was of interest because it determined what kind of spatial knowledge subjects may have: local or global. Step size was related to movement speed and accuracy.

The TWO-FIXED treatment in Table 1 was a conventional virtual environment, in which both eye level and step size were fixed. The CHANGEABLE STEP-SIZE ONLY (STEP-SIZE ONLY) and CHANGEABLE EYE-LEVEL ONLY (EYE-LEVEL ONLY) environments gave subjects the capability to change only the step-size and eye-level parameters respectively. The BOTH cell has an extra comparison (the "+ 1 " part of the " 2 X $2+1$ " design). In the BOTH-COUPLED treatment, the two parameters were coupled and could be changed together. In the BOTH-SEPARATE treatment, two parameters were controlled separately.

Table 1

Treatments in Experiment

\begin{tabular}{|l|l|l|}
\hline & Fixed Eye Level & Changeable Eye Level \\
\hline Fixed Step Size & TWO-FIXED & EYE-LEVEL ONLY \\
\hline Changeable Step Size & STEP-SIZE ONLY & BOTH-COUPLED \\
& & BOTH-SEPARATE \\
\hline
\end{tabular}

\subsection{Subjects}

Recruited through email, twenty paid student subjects (15 male and 5 female) participated in the experiment. All subjects had experience with 3D virtual environments before participating in the study. 


\subsection{Apparatus}

A Dell Precision 340 workstation (1.8 GHz Pentium 4 CPU and 216M memory) was used in the test. The machine had a GeForce2 GTS (32M) graphics card and a 15" monitor with a 1024 X 768 resolution.

Subjects used a keyboard and a mouse to interact with the virtual environments. Four arrow keys were used to control movement. Rotation was controlled by the combination of the Ctrl key and left/right arrow keys. Two parameters of eye-level and step-size were controlled through two sets of keys: press $z$ and $x$ to increase and decrease the eye level respectively, and press $n$ and $m$ to increase and decrease the step size respectively. The mouse was only used to click on buttons to start and end a task. It was not involved in navigation tasks because our pilot tests showed subjects' skills in navigating 3D space with a mouse varied significantly. To avoid the impact of such skill difference on experiment results, we chose to use only keys in navigation control. No subject experienced difficulty in using keys in interaction.

To help subjects better remember the functions of keys, all keys involved in the test were labeled by white tapes with action names, such as eye-level up, step-size down, right turn, and so on. A key-map sticker was placed next to the screen to help users find the functions of keys quickly (Figure 5).

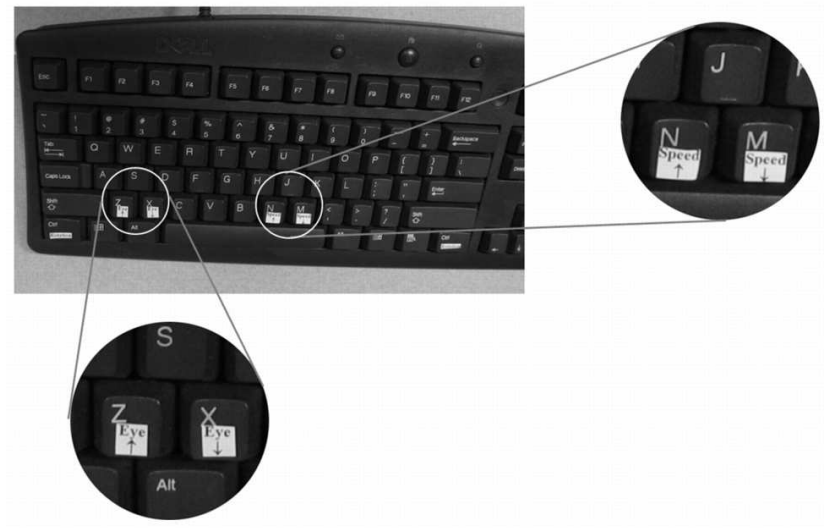

(a): Kay labels.

\subsection{Procedure}

The task was to locate a target object in a 2000m X 2000m square area, a fairly-large area compared with a subject's regular step-size, 0.5m. Subjects were told that the target object, a very small box, was near a target building, which was among four distinctly shaped buildings (square, hexagon, octagon, and circle, as seen in Figure 6) located in each corner of the square area. In the test, the only available information of the target box was the shape 


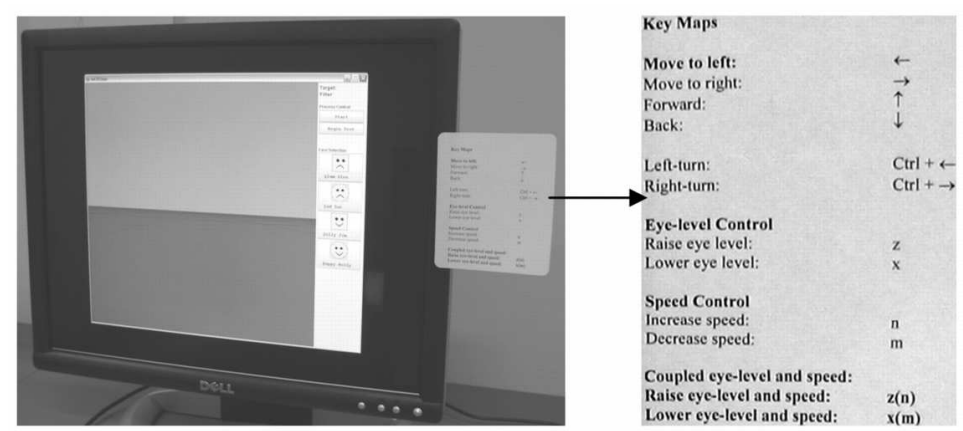

(b): Key-map sticker.

Figure 5. Key labels and Key-map sticker in experiment.

of the target building. Thus, subjects must adopt an exploration approach to navigate through the space to find the building and then the box. Each building had a height of $12 \mathrm{~m}$ and a base of about $80 \mathrm{~m} \mathrm{X} 80 \mathrm{~m}$. On the ground behind each building there was a box $(1 \mathrm{~m} \mathrm{X} 1 \mathrm{~m} \mathrm{X} 1 \mathrm{~m})$ containing a unique text name and smiley face (about $0.5 \mathrm{~m}$ X $0.5 \mathrm{~m}$ ). Figure 6 shows an overview of the area, a view of a box, and a building.

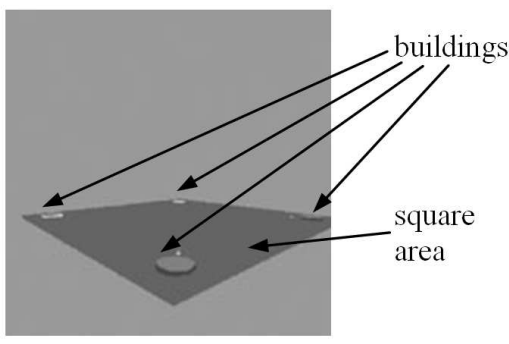

(a)

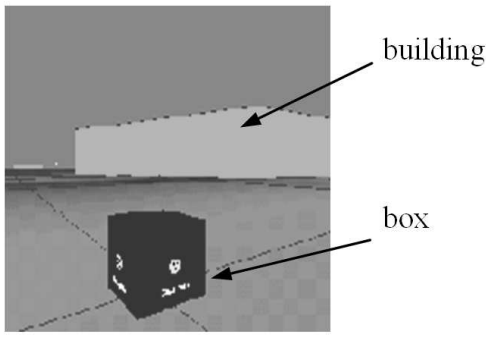

(b)

Figure 6. Views of the test scenes: a) an overview of the square area with four buildings; b) a close view of a goal target.

To complete the task, subjects needed to finish two subtasks: first, to find and approach the target building, and then to identify the target box by reading its text and smiley face. Finding and approaching the target building is a globallevel task requiring global spatial knowledge (e.g., the shape of the target building and its exact location in the area) and fast movement. Identifying the target box has to be done at a local level with detailed information on the box and accurate movement. In the beginning of each test, subjects were in the middle of the square area and were only given the information about the shape of the target building. After finding a target building, subjects clicked a corresponding button. A target box was only visible after a correct target building was identified.

Each subject had an eye-level of $1.68 \mathrm{~m}$ and a step-size of $0.5 \mathrm{~m}$ initially. In treatments that allowed subjects to change the eye-level and step-size, subjects could press appropriate keys to increase or decrease their values. Logarithmic 
functions were used to model these two parameters (Eq. 1 \& 2). Such a logarithmic design follows the design practice seen in multiscale user interfaces, such as $\mathrm{Pad}++$ (Bederson at al., 1994), to give users a constant change rate of view during scaling.

$$
E_{i}=E_{i-1}(1+\alpha)^{k}
$$

Here $E_{i}$ is the eye-level after a key is pressed and $E_{i-1}$ is the eye-level before a key is pressed. $\alpha$ is the scaling factor, which controls the change rate. 0.05 was used in the test. $k$ corresponds the type of change: 1 for increasing eye-level and -1 for decreasing eye-level.

$$
S_{i}=S_{i-1}(1+\alpha)^{p}
$$

Here $S_{i}$ is the step-size after a key is pressed and $S_{i-1}$ is the step-size before a key is pressed. $\alpha$ is the scaling factor, also set to be 0.05 . $p$ corresponds the type of change: 1 for increasing step size and -1 for decreasing it.

In the STEP-SIZE ONLY treatment, $k$ was set to be zero, making a fixed eyelevel. In the EYE-LEVEL ONLY treatment, $p$ was zero to set a fixed step-size. In the BOTH-SEPARATE treatment, both $k$ and $p$ could be modified, but according to different sets of keys: $k$ responding to the $z$ and $x$ keys, and $p$ to the $n$ and $m$ keys. In the BOTH-COUPLED treatment, $k$ and $p$ were coupled together and responded to either set of the keys. Thus, one keystroke could change both eye-level and step-size.

Using a within-subjects design, each subject did the task under five treatments. To counterbalance the possible order effects of these treatments, a Latin square was used to determine different treatment orders. Subjects were randomly assigned to these orders.

The performances were measured by task completion time. In each treatment, subjects practiced until they successfully completed a task similar to the real task in two successive trials. They then performed the real experimental task twice. The average of the results from these two tests was treated as the data. To identify the time consumed for each subtask, subjects were required to report to the system when they correctly identify the target building and began to approach the target box. Subjects were also asked to assess the helpfulness of these five treatments after the test. 


\subsection{Results}

Figure 7 shows the total task completion time. A two-factor ANOVA in four treatments (TWO-FIXED, EYE-LEVEL ONLY, STEP-SIZE ONLY, and BOTH-SEPARATE) showed main effects of both eye level $\left(F_{1,76}=22.61, p<\right.$ $0.0001)$ and step size $\left(F_{1,76}=90.76, p<0.0001\right)$ as well as significant interaction $\left(F_{1,76}=13.64, p=0.0004\right)$. Subjects' performances in the BOTHCOUPLED treatment were better than the SEPARATE treatment. We took a more conservative approach in ANOVA by choosing the BOTH-SEPARATE treatment rather than the BOTH-COUPLED treatment so that the main effects would not be overestimated.

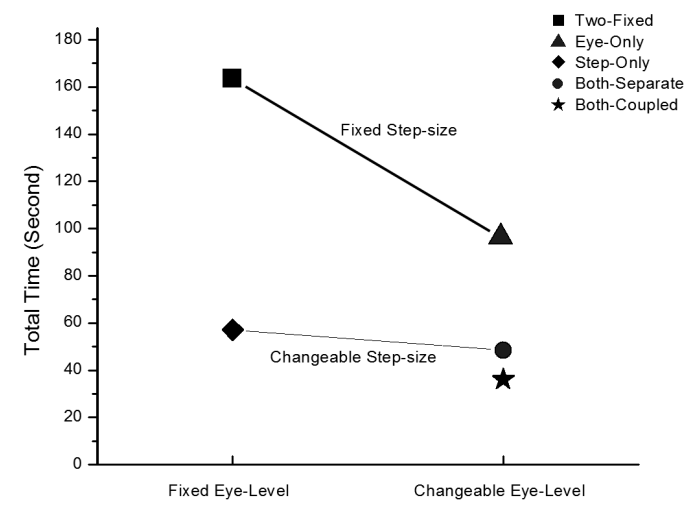

Figure 7. Time comparison for the five treatments.

A two-factor ANOVA of the time consumed for the global-level subtask in the same four treatments showed main effects of both eye level $\left(F_{1,76}=91.56, p<\right.$ $0.0001)$ and step size $\left(F_{1,76}=39.19, p<0.0001\right)$ as well as significant interaction $\left(F_{1,76}=40.59, p<0.0001\right)$ (Figure 8).

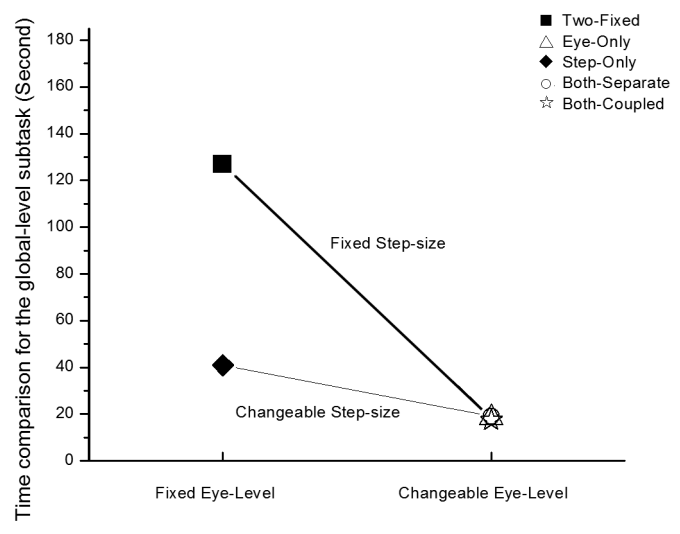

Figure 8. Time comparison for the global-level subtask.

A comparable ANOVA of the time for the local-level subtask also showed main effects of both eye level $\left(F_{1,76}=106.52, p<0.0001\right)$ and step size $\left(F_{1,76}=\right.$ $65.90, p<0.0001)$ as well as significant interaction $\left(F_{1,76}=16.98, p<0.0001\right)$ (Figure 9). 


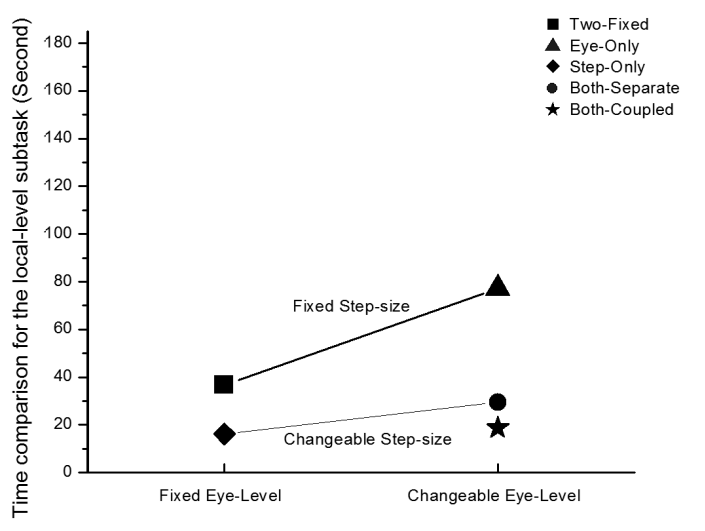

Figure 9. Time comparison for the local-level subtask.

Different interactive techniques in the control of eye-level and step-size were also found to have a significant impact on the completion time of the overall task. The BOTH-COUPLED treatment led to a faster result than the BOTHSEPARATE $\left(t_{19}=4.52, p<0.001\right)$, as seen in Figure 7 .

Such performance difference seems not related to the number of key strokes. Compared with the BOTH-SEPARATE treatment, the BOTH-COUPLED treatment reduces the total number of key strokes for scale and movement speed control by half. Thus, subjects could spend less time pressing keys in the BOTH-COUPLED treatment. However, the difference in total key strokes between these two treatment was not found significant $\left(t_{19}=0.20, p=0.42\right)$.

For the global-level task the difference between these two techniques was not significant $\left(t_{19}=0.37, p=0.72\right.$ ) (Figure 8$)$, but performance in the BOTH-COUPLED treatment was significantly better than that in the BOTHSEPARATE in completing the local-level subtask $\left(t_{19}=6.23, p<0.001\right)$ (Figure 9).

Figure 10 shows the medians of perceived helpfulness of the five treatments, in a seven-level Likert scale (1-extremely unhelpful, 7-extremely helpful). Error bars specify their inter-quartile ranges. Results from Wilcoxon signed ranks tests indicated that the BOTH-COUPLED treatment was perceived significantly better than all other treatments $\left(Z_{\text {вотн-COUPLED vs. Two-FIXeD }}=3.886, p<\right.$

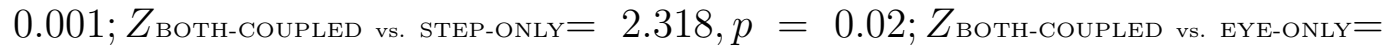
$2.001, p=0.045)$, except the BOTH-SEPARATE treatment $\left(Z_{\text {вотн-COUPLED }}\right.$ vs. вотн-SEPARATE $=1.502, p=0.133)$. 


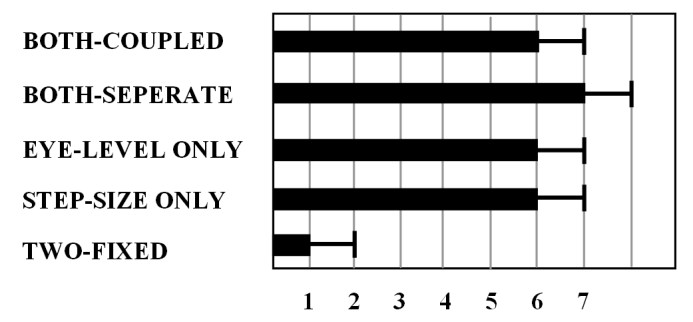

Figure 10. Medians of perceived helpfulness of the five treatments. Error bars indicate their inter-quartile ranges.

\section{Discussion}

\subsection{Navigation as a Multiscale Progressive Process}

Experiment results seem to confirm that a multiscale progressive approach serves users better in exploration-oriented navigation tasks in which users try to locate a target by relying on its descriptive information at different scales, rather than its spatial coordinates. The better performance for the overall task in the BOTH-COUPLED treatment than all other treatments, as seen in Figure 7, indicates that users can benefit from multiscale navigation tools that couple spatial knowledge and movement and progressively refine the granularity of spatial information and movement across scale. Not surprisingly, our results showed it is important to have easy access to spatial information at different levels $\left(F_{1,76}=22.61, p<0.0001\right)$ and flexible control on movement $\left(F_{1,76}=90.76, p<0.0001\right)$ (Figure 7$)$ in navigation tasks. This implies that different spatial knowledge and movement speed and accuracy may help better complete navigation that involves subtasks at different scales.

Such a progressive approach is also perceived better by subjects. Subjects preferred the ability to control eye-level and movement speed across scale. However, statistic result seems to indicate that coupling eye-level and movement was not perceived significantly better than the separate control $\left(Z_{\text {вотн- }}\right.$ COUPLed vs. вотh-Separate $=1.502, p=0.133$ ) (Figure 10), shown in Figure 10. This may be due to two reasons. First, multiscale tools were new to subjects. Most subjects said they were excited about the new design. Such a positive feeling towards multiscale tools may make the difference between the BOTH-COUPLED and BOTH-SEPARATE control less significant. Second, we suspect that the simple test scene may also contribute to the result, because the benefits of BOTH-COUPLE for navigation cannot be fully realized and appreciated in a simple scene. 


\subsubsection{Global-Level Task}

The t-test result for the global-level subtask, $t_{19}=0.37$ and $p=0.72$ (Figure 8 ), seemed to indicate that the coupling of spatial knowledge and movement is less critical to the global-level subtask. Figure 8 shows that as long as subjects were allowed to control eye-level to get an easy access to global spatial information, the global-level subtask could be easily achieved. We suspect that the simplicity of the test scene in the study may contribute to such results, because the simple spatial structure in the scene allowed subjects to quickly glimpse the environment to get a good understanding the space. Moving around to explore the space became unnecessary. Had the test scene been more complicated and demanded more observation of the structure from different perspectives, coupling spatial knowledge and movement might have been more beneficial to navigation.

\subsubsection{Local-Level Task}

The significantly less time spent in the local-level subtask in the BOTHCOUPLED treatment than in the BOTH-SEPARATE $\left(t_{19}=6.23, p<0.001\right)$ (Figure 9) may imply that the coupled design can improve movement accuracy. This may be due to the match between spatial knowledge for planning and that for assessing. Under other treatments, subjects were observed to either have to make great efforts to obtain proper spatial knowledge for assessing action results, such as in the EYE-LEVEL ONLY treatment, or without such knowledge, adopt a time-consuming, trial-and-error strategy, such as in the TWO-FIXED treatment and the STEP-SIZE ONLY treatment.

It should be noted that as Figure 9 shows, those changeable eye-level treatments, except the BOTH-COUPLED one, led to worse performances than their counterpart fixed eye-level treatments in the local-level task. This is due to a longer distance a subject needed to travel in changeable eye-level treatments, which allowed the subject to find a target building afar. In those fixed eye-level treatments, a subject could only find a target building by going to each building and counting its sides. Thus, when a target building was identified, the subject was actually close the final target box with a shorter traveling distance.

Certainly, this long-traveling distance issue could be addressed by integrating tools like logarithmic functions (Mackinlay et al., 1990) or Image-PlaneManipulation (Pierce et al., 1997) as long as a target building is visible. 


\subsection{Limits of the Multiscale Progressive Model}

The multiscale progressive model has some limits. First, this model primarily concerns with navigation in virtual environments in which the destination target is not clearly known and exploration experience is needed. If the target is known, tools like Image-Plane-Manipulation (Pierce et al., 1997) or logarithmic functions (Mackinlay et al., 1990) can be applied. If exploration is not a concern, system-driven navigation or even teleportation can serve users well.

Second, this model does not consider the role of systems in assisting users to find ideal interaction scale levels and identify optimal coupling methods for spatial knowledge and action. Given the complexity of navigation behaviors and the diversity of virtual scenes, users may find it a challenge to figure out what the best strategy could be on their own. Then, users may go back and forth between different scale levels in navigation and scaling control may impost additional cognitive load in navigation, compared with traditional navigation tools. One way to address this issue could be to use "cognitive agents" (Fan et al., 2006) to capture a user's action context and then provide navigation guidance.

Furthermore, the progressive process and the coupling mechanism are primarily designed for navigation in virtual environments, where both locomotion speed and view level can be easily manipulated across scale. Directly applying this process model into the real world may be problematic, given the fact that human beings can hardly perceive objects and act on them beyond our normal interaction scale range (roughly from millimeters to hundred of meters).

Although coupling viewpoint and movement in the real world is not as easy as in virtual environments, it is viable to integrate multiscale technology into digital maps or PDA-based navigation tools (Brachtl et al., 2001) to help people access spatial knowledge in a gradually refined fashion. For example, GPS navigation systems for cars can provide drivers with a list of subtasks at different scale levels, and then show them maps with different levels of details based on what subtasks have been achieved. Compared with turn-byturn driving direction currently available in most GPS navigation systems, a design based on the multiscale progressive model can provide drivers with geographic information that gives them a better understanding of driving goals and the targeted area (Zhang, 2007). 


\section{Conclusion}

Navigation in virtual environments is an inter-disciplinary research issue concerning spatial cognition and technical designs. The landmark-route-survey model from cognitive psychology and geography has had great influence on designs, although there are still unaddressed issues that concern the integration of spatial knowledge and spatial action and their relationship. Virtual environment technologies offer opportunities to deepen our understandings on these issues and to develop and test richer models on the process of applying spatial knowledge in guiding navigation tasks.

This paper proposed a multiscale progressive model on navigation that involves spatial knowledge and spatial action at different scale levels. This model considers not only different kinds of spatial knowledge required in navigation and spatial tasks, but also the association between them. Coupled spatial knowledge and spatial tasks across different scale levels can benefit navigation in large virtual space. An experiment was conducted to study the consequences of such a coupling and easy transition on a two-stage navigation task in a multiscale virtual environment, which allows the coupling and easy transition of spatial knowledge and movement. Results of the experiment indicated that users can improve their performances in navigation involving subtasks at different scale levels if they can couple spatial knowledge and movement together and move them easily across scale. Thus, navigation design in virtual environments should focus on not only helping users access spatial knowledge, but also tailoring the presentation of spatial knowledge according to users' action level and making it easy for users to move between different forms of spatial knowledge.

Our future research efforts will be made to advance the understanding of the benefits of coupling spatial knowledge and action for spatial cognition and related spatial actions. In this study, we focused on the efficiency of navigation activities. We are also interested in the impact of the coupling on spatial cognition in complex environments, such as the acquisition of spatial information at different levels and the integration of spatial information across scale into coherent cognitive maps.

The easy control over spatial knowledge also provides opportunities to study the complexity of the construction of spatial knowledge by going beyond the traditional views of spatial knowledge as sequential and leveled processes. It has been argued that the construction of spatial knowledge may involve various forms of spatial information and various techniques (Montello, 2001; Tversky, 1993; Hirtle \& Hudson, 1991; Presson \& Montello, 1988). In mVEs, users can easily control what spatial knowledge should be present and in what sequence (e.g., in a global-to-local direction or a local-to-global direction). 
Such flexibility in interaction with multiscale virtual space allows us to observe how different kinds of spatial knowledge and different techniques to access spatial knowledge may affect the understanding of space. Thus, multiscale technology could be a valuable tool to deepen our understanding of users' spatial behaviors in virtual environments, and then shed some light on spatial knowledge acquisition in the real world.

One challenge in using multiscale technology in the real world is to create multiscale maps that can provide people with geographic information that better suits people's navigation tasks at different scale levels. Although we can find maps at different scales, our understanding of the cognitive implications of maps at different scales for human actions is still poor (Mark et al., 1999). In multiscale user interfaces, the same structure can be displayed with different representations at different scales. These representations differ from each other not only in geometric size, but also in semantic features. Such scale-based semantic representations (Perlin \& Fox, 1991; Bederson \& Hollan, 1994; Zhang \& Furnas, 2005a) can be a good tool to visualize spatial structures with different models that concern different conceptual abstractions of spatial information (Remolina et al., 1999; Timpf, 1999), but it is still unclear how such multiscale representations can benefit human navigation activities cognitively in the real world. We are also interested in studying this issue.

\section{Acknowledgement}

The author like to thank George Furnas for his valuable help on this research and anonymous reviewers for their constructive comments. This research was funded in part by Microsoft Research.

\section{References}

Ahl, V., \& Allen, T. (1996). Hierarchy Theory: A Vision, Vocabulary and Epistemology. Columbia University Press, New York.

Albrecht, J., \& Car, A. (1999). GIS Analysis for Scale-Sensitive Environmental Modelling Based on Hierarchy Theory. In Dikau, R. \& Saurer, H. (Eds.) GIS for Earth Surface Systems, pp. 1-23.

Alexander, C., Ishikawa, S., \& Silverstein, M. (1977). A Pattern Language. Oxford University Press.

Bagrow, L. (1985). History of Cartography. Precedent Publishers, Chicago.

Barkowsky, T., \& Freksa, C. (1997). Cognitive Requirements on Making and Interpreting Maps. In Hirtle, S., \& Frank, A. (Eds.), Spatial Information 
Theory: A Theoretical Basis for GIS. Proceedings of COSIT 97, pp. 347361, Springer, Berlin.

Bederson, B., \& Hollan J. (1994). Pad++: A Zooming Graphical Interface for Exploring Alternate Interface Physics. Proceedings of the ACM Symposium of User Interface Software and Technology (UIST '94), pp.17-26.

Bowman, D., Koller, D., \& Hodges, L. (1997) Travel in Immersive Virtual Environments: An Evaluation of Viewpoint Motion Control Techniques. Proceedings of the VR Annual International Symposium, pp. 45-52.

Brachtl, M., Šlajs, J. \& Slavík, P. (2001). PDA Based Navigation System for a 3D Environment. Computers \& Graphics, 25(4), pp. 627-634

Chase, W. C. (1983). Spatial Representations of Taxi Drivers. In Rogers, D., \& Sloboda, J. (eds.) Acquisition of Symbolic Skills. Plenum Press, New York, 1983, pp. 391-405.

Chen, J. L., \& Stanney, K. M. (1999). A Theoretical Model of Wayfinding in Virtual Environments: Proposed Strategies for Navigational Aiding. Presence: Teleoperators and Virtual Environments 8 (6), pp. 671-685.

Cohen, S. L., \& Cohen, R. (1985). The Role of Activity in Spatial Cognition. In R. Cohen (Ed.), The Development of Spatial Cognition, pp. 199-223. Hillsdale, NJ: Lawrence Erlbaum.

Colle, H., \& Reid, G. (1998). The Room Effect: Metric Spatial Knowledge of Local and Separated Regions. Presence: Teleoperators and Virtual Environments, 7, 116-128.

Combs, T., \& Bederson, B. B. (1999). Does Zooming Improve Image Browsing? Proceedings of the Fourth ACM International Conference on Digital Libraries, pp. 130-137.

Darken, R. P., \& Sibert, J. (1996). Navigating Large Virtual Spaces. International Journal of Human-Computer Interaction, 8 (1), pp. 49-71.

Darken, R. P., \& Cevik, H. (1999). Map Usage in Virtual Environments: Orientation Issues. Proceedings of IEEE Virtual Reality '99, pp. 133-140.

Downs, R., \& Stea, D. (1973). Image and Environment: Cognitive Mapping and Spatial Behavior. Chicago: Aldine.

Elvins, T., Nadeau, D., \& Kirsh, D. (1997). Worldlets-3D Thumbnails for Wayfinding in Virtual Environments. Proceedings of the ACM Symposium of User Interface Software And Technology (UIST '97), pp. 21-30.

Evans, G.W., \& Pezdek, K. (1980). Cognitive Mapping: Knowledge of RealWorld Distance and Location Information. Journal of Experimental Psychology: Human Learning and Memory, vol. 6, no. 1, pp. 13-24.

Fan, X., Sun, B, Sun, S., McNeese, M., \& Yen, J. (2006) RPD-Enabled Agents Teaming with Humans for Multi-Context Decision Making. In Proceedings of the Fifth International Joint Conference on Autonomous Agents and Multi Agent Systems (AAMAS'06), pp. 34-41

Furnas, G. W., \& Bederson B. B. (1995). Space-Scale Diagrams: Understanding Multiscale Interfaces Papers: Navigating and Scaling in 2D Space. Proceedings of the ACM Conference on Human Factors in Computing System (CHI '95), pp. 234-241. 
Ghosh, P., \& Shneiderman, B. (1999) Zoom-Only vs. Overview-Detail Pair: A Study in Browsing Techniques as Applied to Patient Histories. University of Maryland Technical Report CS-TR-4028.

Golledge, R. (1999). Human Wayfinding and Cognitive Maps. In Golledge, R. (Ed.), Wayfinding Behavior: Cognitive Maps and Other Spatial Processes, pp. 5-45, Johns Hopkins University Press, Baltimore.

Guiard, Y., Beaudouin-Lafon, M., \& Mottet, D. (1999). Navigation as Multiscale Pointing: Extending Fitts' Model to Very High Precision Tasks. Proceedings of the ACM Conference on Human Factors in Computing System (CHI '99), pp. 450-457.

Hanson, A., Wernert, E., \& Hughes, S. (1997). Constrained Navigation Environments. Scientific Visualization: Dagstuhl '97 Proceedings, pp. 95-104.

Hart R., \& Moore, G. (1973). The Development of Spatial Cognition: A Review. In Stea, B., \& Downs, R. (Eds.), Image and Environment, pp. 226-234. University of Chicago Press, Chicago.

Hirtle, S. C., \& Hudson, J. H. (1991). The Acquisition of Spatial Knowledge. Journal of Environmental Psychology, 11, pp. 335-345.

Hommel, B., Gehrke, J., \& Knuf, L. (2000). Hierarchical Coding in the Perception and Memory of Spatial Layouts. Psychological Research, 64, pp. $1-10$.

Hornbæk, K., Bederson, B., \& Plaisant, C. (2002). Navigation Patterns and Usability of Zoomable User Interfaces with and without Overview. Transaction of Computer Human Interaction, 9, pp. 362-389.

Hutchins, E. (1995). Cognition in the Wild. Cambridge, MA: MIT Press.

Jul, S., \& Furnas, G. W. (1997). Navigation in Electronic Worlds. SIGCHI Bulletin, 29 (4), pp. 44-49.

Jul, S., \& Furnas, G. W. (1998). Critical Zones in Desert Fog: Aids to Multiscale. Proceedings of the ACM Symposium of User Interface Software And Technology (UIST '98), pp. 97-106.

Kopper, R., Ni, T., Bowman, D., \& Pinho, R. (2006) Design and Evaluation of Navigation Techniques for Multiscale Virtual Environments. Proceedings of the IEEE Virtual Reality 2006, pp. 181-189.

Kosslyn, S. M., Ball, T. M., \& Reiser, B. J. (1978). Visual Images Preserve Metric Spatial Information: Evidence from Studies of Image Scanning. Journal of Experimental Psychology: Human Perception and Performance, 4, pp. 47-60.

Lam, N., \& Quattrochi, D. A. (1992). On the Issues of Scale, Resolution, and Fractal Analysis in the Mapping Sciences. The Professional Geographer, 44, pp. 88-98.

LaViola, J., Feliz, D., Keefe, D., \& Zeleznick, R. (2001). Hands-Free MultiScale Navigation in Virtual Environments. Proceedings of the Symposium on Interactive 3D Graphics, pp. 9-15.

Leigh, J., \& Johnson, A. (1996). Supporting Transcontinental Collaborative Work in Persistent Virtual Environments. In IEEE Computer Graphics and Applications, 16(4), pp. 47-51. 
Levine, D., Warach, J., \& Farah, M. (1985). Two Visual Systems in Mental Imagery: Dissociation of "What" and "Where" in Imagery Disorders Due to Bilateral Posterior Cerebral Lesions. Neurology, 35, pp. 1010-1018.

Liben, L. S. (2001). Thinking through Maps. In Gattis, M. (Ed.), Spatial Schemas and Abstract Thought, pp. 44-77, MIT Press, Cambridge.

Lynch, K. (1960). Image of the City, MIT Press, Cambridge.

Mackinlay, J., Card, S., \& Roberston, G. (1990). Rapid Controlled Movement through a Virtual 3D Workspace. Computer Graphics, 24(4), pp. 171-166.

Mapes, D. P., \& Moshell, J. M. (1995). A Two-Handed Interface for Object Manipulation in Virtual Environments. Presence: Teleoperators and Virtual Environments, 4(4), pp.403-416.

Mark, D. M., Freksa, C., Hirtle, S. C., Lloyd, R., \& Tversky, B. (1999). Cognitive models of geographical space. International Journal of Geographical Information Science, 13, pp. 747-774.

May, M., Péruch, P., \& Savoyant, A. (1995). Navigating in a Virtual Environment With Map-Acquired Knowledge: Encoding and Alignment Effects. Ecological Psychology, 7(1), pp. 21-36.

McNamara, T. P., Hardy, J. K., \& Hirtle, S. C. (1989). Subjective Hierarchies in Spatial Memory. Journal of Experimental Psychology: Learning, Memory and Cognition, 15, pp. 211-227.

Mine, M., Brooks, F., \& Sequin, C. (1997). Moving Objects in Space: Exploiting Proprioception in Virtual-Environment Interaction. Proceedings of the ACM SIGGRAPH '97, pp. 19-26.

Montello, D. R. (1993). Scale and Multiple Psychologies of Space. In Frank, A. U. \& Campari, I. (Eds.). Spatial Information Theory: A Theoretical Basis for GIS, pp. 312-321.

Montello, D. R. (2001). Spatial Cognition. In Smelser, N., \& Baltes, P. (Eds.), International Encyclopedia of the Social \& Behavioral Sciences, pp. 1477114775. Oxford: Pergamon Press.

Newcombe, N., \& Huttenlocher, J. (2000). Making Space: The Development of Spatial Representation and Reasoning, MIT Press, Cambridge.

Pereira, G. (2002). A Typology of Spatial and Temporal Scale Relations. Geographical Analysis 34(1), pp. 21-33

Perlin, K., \& Fox D. (1993). Pad: An Alternative Approach to the Computer Interface. Proceedings of the ACM SIGGRAPH '93, pp. 57-64.

Péruch, P., Vercher, J. L., \& Gauthier, G. M. (1995). Acquisition of Spatial Knowledge through Visual Exploration of Simulated Environments. Ecological Psychology, 7 (1), pp. 1-20.

Piaget, J., \& Inhelder, B. (1967). The Child's Conception of Space. New York: Norton, 1967.

Pierce, J., \& Pausch, R. (2004). Navigation with Place Representations and Visible Landmarks, Proceedings of VR 2004, pp. 173-180.

Pierce, J., Forsberg, A., Conway, M., Hong, S., Zeleznik, R., \& Mine, M. (1997). Image Plane Interaction Techniques in 3D Immersive Environments. Proceedings of the Symposium on Interactive 3D Graphics, pp. 39-43. 
Presson, C., \& Montello, D. (1988). Points of Reference in Spatial Cognition: Stalking the Elusive Landmark. British Journal of Developmental Psychology, 6, pp. 378-381.

Puppo, E., \& Scopigno, R. (1997). Simplification, LOD, Multiresolution: Principles and Applications. Proceedings of EUROGRAPHICS, 16 (3).

Reitsma, F. \& Bittner, T. (2003). Scale in Object and Process Ontologies. In Kuhn, W., Worboys, M. F. \& Timpf, S. (Eds.) Spatial Information Theory: Foundations of Geographic Information Science. Proceedings of COSIT'03, pp. 13-30, Springer-Verlag, Germany.

Robinett, W., \& Holloway, R. (1992). Implementation of Flying, Scaling and Grabbing in Virtual Worlds. Proceedings of the Symposium on Interactive 3D Graphics, pp. 189-192.

Ruddle, R., Payne, S., \& Jones, D. (1997). Navigating Buildings in "DeskTop" Virtual Environments: Experimental Investigations Using Extended Navigational Experience. Journal of Experimental Psychology: Applied, 3 (2), pp. 143-159.

Siegel, A., \& White, S. (1975). The Development of Spatial Representations of Large-Scale Environments. In Reese, H. (Ed.): Advances in Child Development and Behavior, 10, pp. 9-55, Academic Press, New York.

Stevens, A. \& Coupe, P. (1978). Distortions in Judged Spatial Relations. Cognitive Psychology 10, pp. 422-437.

Stoakley, R., Conway, M., \& Pausch, R. (1995). Virtual Reality on a WIM: Interactive Worlds in Miniature. Proceedings of the ACM Conference on Human Factors in Computing System (CHI '95), pp. 265-272.

Tan, D., Robertson, G., \& Czerwinski, M. (2001). Exploring 3D Navigation: Combining Speed-Coupled Flying with Orbiting. Proceedings of the ACM Conference on Human Factors in Computing System (CHI '2001), pp. 418425.

Thorndyke, P., \& Hayes-Roth, B. (1982). Differences in Spatial Knowledge Acquired from Maps and Navigation. Cognitive Psychology, 14, pp. 560589.

Timpf, S. (1999). Abstraction, Levels of Detail, and Hierarchies in Map Series. In Freksa, C, Mark, D. M. (Eds.), Spatial Information Theory: Cognitive and Computational Foundations of Geographic Information Science. Proceedings of COSIT '99, pp. 125-139, Springer-Verlag, Germany.

Timpf, S. \& Kuhn, W. (2003) Granularity Transformations in Wayfinding. In Freksa, C., Brauer, W., Habel, C., \& Wender, K. (Eds.), Spatial Cognition III: Routes and Navigation, Human Memory and Learning, pp. 77-88.

Tolman, E.C. (1948). Cognitive Maps in Rats and Men. Psychological Review, 55, pp. 189-208.

Tversky, B. (1993). Cognitive Maps, Cognitive Collages, and Spatial Mental Models. In Frank, A., Campari, I. (Eds.), Spatial Information Theory: A Theoretical Basis for GIS. Proceedings of COSIT '93, pp. 14-24, SpringerVerlag, Germany.

Vinson, N. G. (1999). Design Guidelines for Landmarks to Support Navigation 
in Virtual Environments. Proceedings of the ACM Conference on Human Factors in Computing System (CHI '99), pp. 278-285.

Ware, C., \& Fleet, D. (1997). Context Sensitive Flying Interface. Proceedings of Symposium on Interactive 3D Graphics, pp. 127-130.

Wilson, P. N., Foreman, N., \& Tlauka, M. (1997). Transfer of Spatial Information from a Virtual to a Real Environment. Human Factors, 39 (4), pp. 526-531.

Witmer, B. G., Bailey, J. H., Knerr, B. W., \& Parsons, K.C. (1996). Virtual Spaces and Real World Places: Transfer of Route Knowledge. International Journal of Human-Computer Studies, 45, pp. 413-428.

Zhang, X. (2007). M²S Maps: Supporting Real-World Navigation with Mobile VR. Virtual Reality. vol. 11, no. 2-3, pp. 161-173.

Zhang, X., \& Furnas, G. W. (2005a). mCVEs: Using Cross-Scale Collaboration to Support User Interaction with Multiscale Structures. Presence: Teleoperators and Virtual Environments, 14(1), pp. 31-46.

Zhang, X., \& Furnas, G. W. (2005b). Multiscale Space and Multiscale Place. In Davenport, E. \& Turner, P. (Eds.): Space, Spatiality and Technologies. Springer, pp. 261-280. 